\title{
Zementaugmentierte Pedikelschrauben
}

Thomas R. Blattert

\section{Einleitung}

Die typische Patientenstruktur in der Wirbelsäulenchirurgie zeigt seit Jahren eine deutliche Tendenz hinsichtlich einer Zunahme der Patienten mit reduzierter Knochenqualität. Dies erklärt sich zum einen aus der zunehmenden Alterung der Bevölkerung und der damit verbundenen typischen osteopenischen bzw. osteoporotischen Wirbelkörperbeschaffenheit. Andererseits gibt es jedoch neben der altersbedingten Osteoporose zahlreiche weitere Gründe, die zu einer Reduktion der Knochenqualität führen können.

Was auch immer der Grund für reduzierte Knochenqualität im Einzelnen ist - alle diese Situationen stellen eine besondere Herausforderung an die Wirbelsäulenchirurgie dar. Rasch nachlassende Primärstabilität durch sekundäre Schraubenlockerung oder sekundäres Auswandern der Schrauben aus dem Wirbelkörper sind eine Folge davon. Aber auch bereits der Versuch einer instrumentellen Reposition intraoperativ kann zu initialer Schraubenlockerung führen.

Aufgrund dieses zunehmend in den Fokus der Wirbelsäulenchirurgen rückenden Problems sind in den vergangenen Jahren verschiedene Techniken entwickelt worden, die den initialen Halt von Pedikelschrauben im strukturgeschwächten Knochen erhöhen. Eine der am häufigsten durchgeführten Techniken ist hierbei sicherlich die Zementaugmentation der Pedikelschraube selbst [1-4]. Weitere beschriebene Techniken sind z. B. in situ expandierbare Pedikelschrauben. Hydroxylapatitbeschichtete Schrauben sind der Gruppe der erst sekundär einen zunehmenden Schraubenhalt entwickelnden Systeme zuzuordnen, die allerdings initial noch keine vergrößerte Haltekraft aufweisen.

Dabei ist das Versagen von Pedikelschrauben im osteoporotischen Wirbelkörper eine seit Langem bekannte und in der Literatur beschriebene Tatsache. Das Nichtbeachten dieser potenziellen Gefahr führt durch das mögliche Sekundärversagen der Instrumentierung zu einem hohen Revisionsbedarf, dessen technische Anforderungen meist deutlich über der Initial-OP liegen ( $\bullet$ Abb. 1). So kann in der einfachen Frakturbehandlung aus einer zunächst möglichen perkutanen Hybridversorgung mit zementaugmentiertem kurzstreckigem Fixateur interne und Ze- mentaugmentation des gebrochenen Indexwirbels bei einem Verzicht auf die Zementaugmentation der Pedikelschrauben und einem subsequenten sekundären Korrekturverlust die Notwendigkeit einer umfangreichen dorsoventralen Rekonstruktion - meist langstreckig - der nun entstandenen posttraumatischen Deformität erwachsen.

\section{Länge der Instrumentierung}

Für die Ausdehnung des Konstrukts gibt es mehrere Überlegungen:

\section{Merke}

Bei längerstreckigen Instrumentierungen sollte grundsätzlich das Pedikelschraubenpaar am kranialen und am kaudalen Ende der Instrumentierung zementaugmentiert werden.

Im dazwischen liegenden Bereich kann u.U. auf eine Zementaugmentation jeder einzelnen Schraube verzichtet werden. Die Länge des Konstrukts hängt somit sowohl von der zugrunde liegenden Pathologie als auch vom Erfolg einer homogenen Zementinjektion um das jeweils am kranialen und kaudalen Ende gelegene Pedikelschrau-
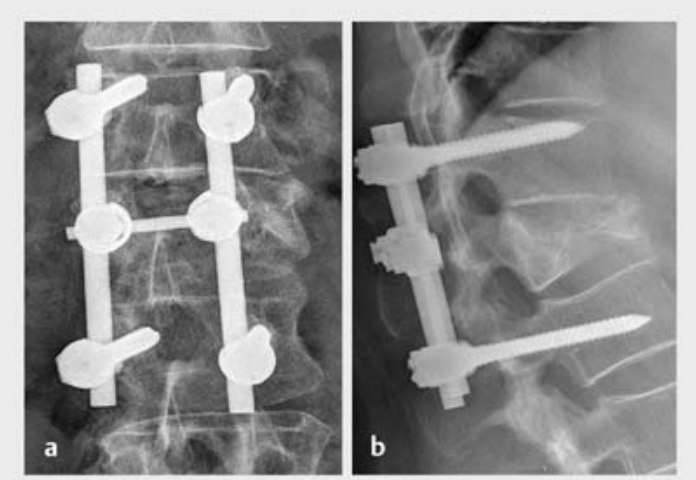

- Abb. 1 64-jährige Patientin: Konventionelle Röntgenaufnahmen in der a.-p. (a) und seitlichen (b) Ebene 3 Monate nach kurzstreckiger nicht zementaugmentierter Instrumentierung einer L-I-Fraktur im strukturschwachen Knochen mit sekundärem Korrekturverlust und segmentaler Rekyphosierung. 

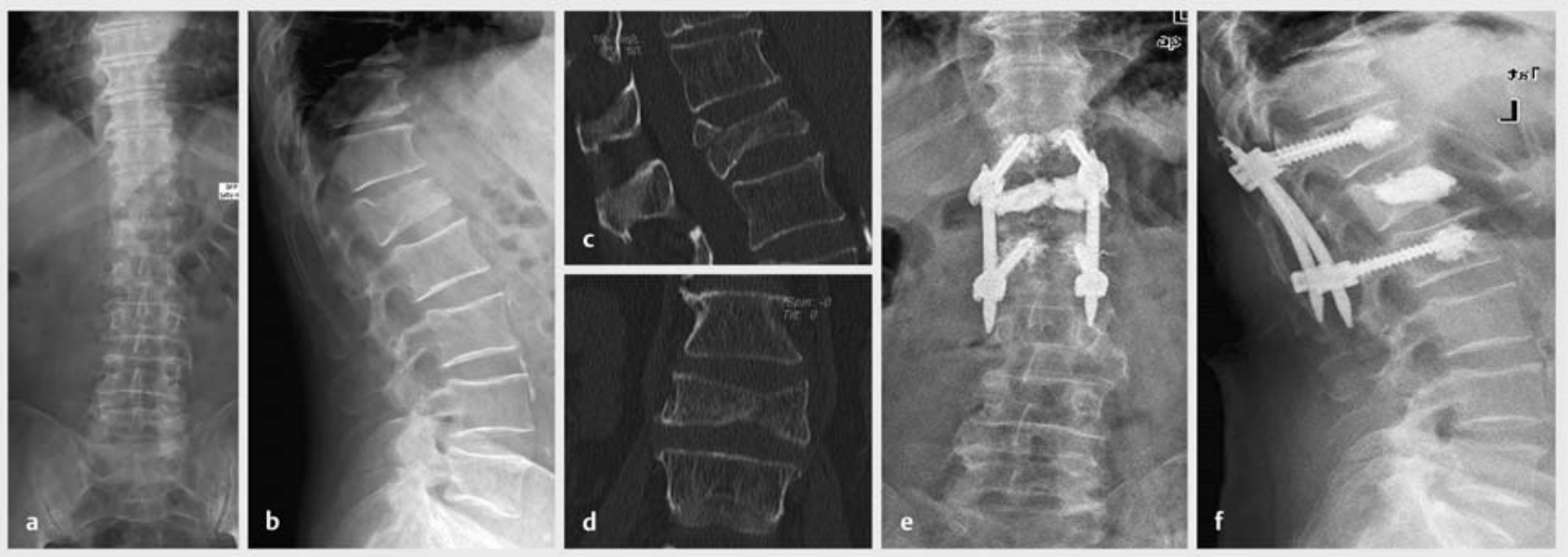

- Abb. 2 72-jähriger Patient: Konventionelle Röntgenaufnahme in der a.-p. (a) und seitlichen (b) Ebene sowie sagittale (c) und koronare (d) CTRekonstruktion einer L-II-Fraktur Typ OF3. Konventionelle Röntgenaufnahmen in der a.-p. (e) und seitlichen (f) Ebene 12 Monate nach kurzstreckiger zementaugmentierter Instrumentierung und Zementaugmentation des Indexwirbels im strukturschwachen Knochen ohne Zeichen einer Materiallockerung oder eines sekundären Korrekturverlusts.

benpaar ab. Sollte es hier zu einem technischen Problem kommen, muss die Erweiterung der Instrumentierung um ein entsprechendes weiteres Schraubenpaar erwogen werden.

Generell kann bei einer einfachen, operativ zu behandelnden osteoporotischen Wirbelkörperfraktur, z. B. einer Fraktur im thorakolumbalen Bereich mit der Klassifikation OF3 [5], eine kurzstreckige Instrumentierung mit nur einem Schraubenpaar oberhalb und einem Schraubenpaar unterhalb des gebrochenen Indexwirbels völlig ausreichend sein $(\checkmark$ Abb. 2 e bis f). Andererseits muss bei biomechanisch anspruchsvolleren Verletzungen wie z.B. einer OF4- und OF5-Verletzung [5] eine längerstreckige Instrumentierung mit kranial 2 bis 3 und kaudal ebenfalls 2 bis 3 Pedikelschraubenpaaren erwogen werden [6]. Insofern lassen sich für die Länge der Instrumentierung keine Grundsatzregeln aufstellen, vielmehr ist es eine individuelle Entscheidung im Einzelfall, die die Konstruktlänge bestimmt.

Merke

Es ist in jedem Fall erforderlich, im Rahmen der präoperativen Aufklärung den Patienten darauf aufmerksam zu machen, dass u. U. längerstreckige Konstrukte erforderlich werden können.

\section{Schraubendesign und Zementiertechniken}

Es gibt grundsätzlich 2 verschiedene Schraubentypen, die unterschiedliche Zementiertechniken notwendig machen: zum einen ist dies der solide, zum anderen der fenestrierte Schraubentyp.
Für den soliden Schraubentyp gibt es zum einen die Möglichkeit des Vorbefüllens des Wirbelkörpers in der sog. Vertebroplastietechnik und die anschließende Insertion der Pedikelschraube in den Wirbelkörper, noch bevor der Zement polymerisiert. Zum anderen gibt es die Möglichkeit, zunächst ein vorgeschnittenes Schraubenlager anzufertigen, z.B. mit einem Gewindeschneider, und anschließend dieses mit Zement zu befüllen. Die solide Schraube wird nun, ebenfalls noch vor Polymerisation des Zements, in das Schraubenlager inseriert.

Beim fenestrierten Schraubentyp wird die Schraube in den nativen Wirbelkörper in ihre definitive Endstellung eingebracht und der Zement anschließend über die Schraubenkanülierung und die Schraubenfenestrierungen in den Wirbelkörper injiziert.

Merke

Aus unserer Sicht hat jede Zementiertechnik eine ähnliche Lernkurve; wir empfehlen, die Technik durchzuführen, die vor Ort Standard ist und komplikationsarm durchgeführt wird, ohne eine bestimmte Technik zu bevorzugen. In unserem Haus findet die Technik mit Verwendung des fenestrierten Schraubentyps bei Weitem am häufigsten Anwendung.

\section{Operationstechnik}

Grundsätzlich können zementaugmentierte Pedikelschrauben sowohl in offener Technik als auch in perkutaner Technik implantiert werden. Im Bereich der Alterschirurgie der Wirbelsäule setzt sich aufgrund des reduzierten operativen Traumas und der häufigen Multimorbidität 


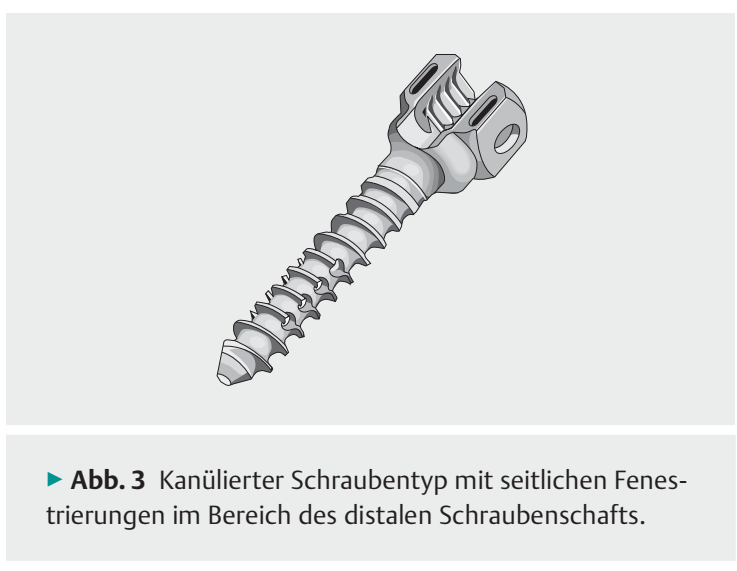

der Patienten zunehmend die perkutane Instrumentierungstechnik durch. Die entsprechenden Pedikelschrauben weisen neben der vorhandenen Kanülierung zur perkutanen Insertion auch Fenestrierungen ( $\boldsymbol{A} \mathbf{A b} \mathbf{b}$. $\mathbf{3}$ ) in verschiedenen, anbieterspezifischen Designs auf.

Zusätzlich zur Standardoperationstechnik für perkutane Pedikelschraubensysteme müssen bei der zementaugmentierten Variante einige weitere intraoperative Details beachtet werden: So sollte unbedingt vermieden werden, den Wirbelkörper mit dem Kirschner-Draht ventral zu perforieren, um das Risiko einer anschließenden Zementextrusion nach ventral zu vermeiden. Gleichzeitig sollte die Pedikelschraube in ihrer Länge nicht bis unmittelbar an die vordere Wirbelkörperbegrenzung herangeführt werden, da dann die Pedikelschraubenspitze selbst nicht mehr hinreichend von der nun zu injizierenden Zementmasse umhüllt werden kann. Aus dem gleichen Grund ist eine unmittelbar deck- oder grundplattennahe Insertion der Pedikelschraube zu vermeiden: Auch in diesem Fall wird eine homogene sphärische Zementverteilung um die Pedikelschraubenspitze herum behindert [7].

\section{Merke \\ Wir empfehlen generell die Verwendung von 1,5 ml Zement für lumbale bzw. die Verwendung von 1,2 ml Zement für thorakale Pedikelschrauben, wobei das Totraumvolumen, welches im Pedikelschrauben- schaft verbleibt, selbstverständlich berücksichtigt werden muss.}

Wenn zusätzlich zur zementaugmentierten Instrumentierung eine Wirbelkörperzementaugmentation, z. B. im Rahmen einer Hybridstabilisierung einer Wirbelkörperfraktur, durchgeführt werden soll, stellt sich häufig die Frage, in welcher Reihenfolge die einzelnen Schritte bewerkstelligt werden sollten. Beendet man erst die Instrumentierung einschließlich der Insertion des Längsträgers, kann dieser u. U. die Sicht auf die Pedikel und damit die Insertion der Injektionskanüle im gebrochenen Indexwir- bel behindern. Andererseits nimmt man sich bei umgekehrter Reihenfolge u. U. die Möglichkeit einer verbesserten Reposition über die Instrumentierung.

Wir sind daher für die Frakturversorgung generell zu folgendem Verfahren übergegangen: Nach Lagerung auf dem Operationstisch erfolgt in der seitlichen Bildverstärkereinstellung die Evaluation der Spontanreposition. Ist diese wie gewünscht, kann zunächst der gebrochene Wirbelkörper zementaugmentiert werden und anschließend die Instrumentierung durchgeführt werden ( $\triangleright$ Abb.4). Ist andererseits die Spontanreposition durch Lagerung in hyperlordotischer Position auf dem Operationstisch nicht ausreichend, wird zunächst die Instrumentierung durchgeführt und in deren Rahmen eine Optimierung der Reposition erreicht. Anschließend wird die Wirbelkörperzementaugmentation durchgeführt. Sollte tatsächlich der Längsträger die unmittelbare Sicht auf den Pedikel des gebrochenen Indexwirbels verlegen, genügt häufig bereits eine geringe Lateralrotation des Bildverstärkers, um die Überlagerung beider Strukturen aufzuheben $[8,9]$.

Die Frage der Zementwahl für die Augmentation von Pedikelschrauben beantworten wir eindeutig mit der Verwendung von PMMA-basierten Zementen (PMMA: Polymethylmethacrylat). Die bislang zur Verfügung stehenden alternativen Materialien auf Kalzium-Phosphat-Basis bzw. Hydroxylapatitbasis haben nicht die notwendigen biomechanischen Eigenschaften, um den im Bereich der Schraubenspitze wirkenden Kräften dauerhaft standzuhalten. Hier ist insbesondere die Scher- und Extensionsfestigkeit dieser alternativen Zementmaterialien nicht ausreichend, sodass wir deutlich vor deren Verwendung zur Pedikelschraubenaugmentation warnen.

\section{Eigene Ergebnisse}

In einer eigenen Traumastudie bei Frakturen jeweils einer Etage des Typs OF3 im Bereich von Th XI bis L V wurden die folgenden Einschlusskriterien definiert: 1. Die präoperativ durchgeführte Kernspintomografie zeigt keinen Hinweis auf eine Verletzung der angrenzenden Bandscheiben. 2. Der T-Score im präoperativ durchgeführten DXA-Scan liegt bei höchstens $-2,5$. Insgesamt wurden 52 Patienten mit 208 augmentierten Pedikelschrauben eingeschlossen. Das Durchschnittsalter lag bei 74 Jahren (60-92). Der durchschnittliche T-Score lag bei $-2,7$ $(-3,1--2,5)$. Insgesamt zeigten sich zum Nachuntersuchungszeitraum mindestens 6 Monate postoperativ 2 Schraubenlockerungen. Die Ursache war in beiden Fällen eine technisch nicht optimal gelungene Zementinjektion mit asymmetrischer Zementverteilungsgeometrie um die Schraubenspitze herum. Die Leckagerate lag mit 41 von 208 Pedikelschrauben bei 19,7\%, wobei in 40 Fällen eine Leckage lateral oder ventral des Wirbelkörpers zu sehen war. In einem Fall handelte es sich um eine epidu- 

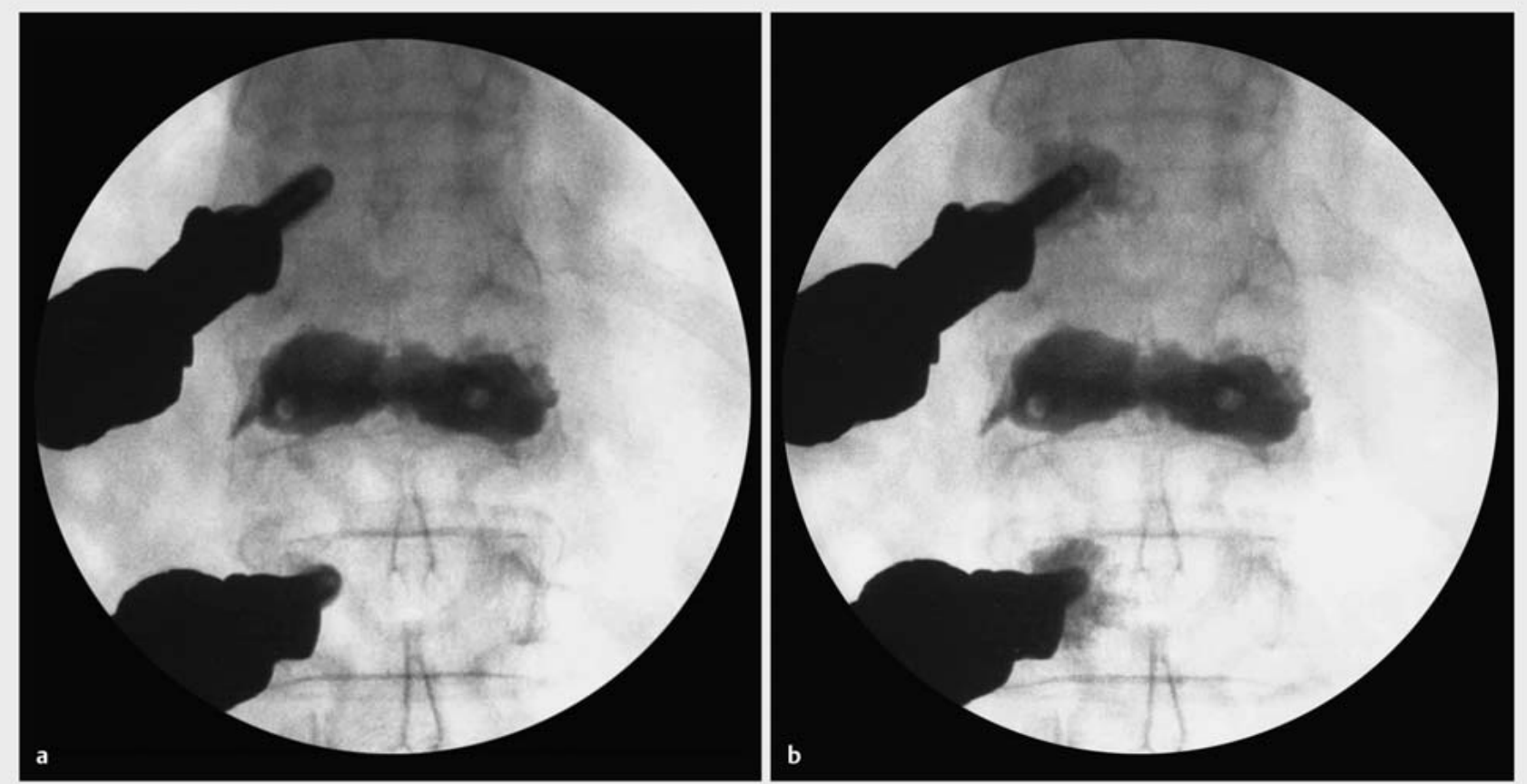

Abb. 4 77-jährige Patientin: intraoperative Bildverstärkeransichten des thorakolumbalen Übergangs in der a.-p. Ebene nach Zementaugmentation des frakturierten Indexwirbelkörpers L I im strukturschwachen Knochen. Die linksseitige Instrumentierung mit kanülierten fenestrierten Pedikelschrauben ist bereits durchgeführt (a), nun erfolgt die Injektion von jeweils 1,5 ml PMMA-Zement über den Schraubenschaft durch die Fenestrierungen in den Wirbelkörper (b).

rale Leckage. Kein Patient erlitt neurologische Komplikationen [10].

\section{Problem Zementleckage}

Die Zementleckage ist ein verfahrensimmanentes technisches Problem [11, 12]. Aufgrund der Grundsätzlichkeit des Problems ist es unbedingt erforderlich, jeden Patienten über die Komplikationsmöglichkeit der Zementleckage aufzuklären. Insgesamt sind neben extravertebralen und epiduralen Zementaustritten auch endovaskuläre Leckagen entlang der drainierenden Segmentalgefäße sowie intradiskale Zementleckagen möglich.

\section{Merke}

Die durchschnittliche Zementleckagerate liegt sowohl in unserem eigenen Patientengut als auch in der zur Verfügung stehenden Literatur bei ca. $20 \%$.

Diese Leckagerate erscheint hoch, wobei Studien ohne postoperative CT-Untersuchung sicherlich das Problem einer deutlich zu niedrigen Erkennungs- und damit Komplikationsrate haben. Andererseits bleibt nahezu jede Zementleckage klinisch inapparent. Aus diesem Grund ist es entscheidend, die intraoperativ erkannte Zementleckage hinsichtlich ihrer möglichen klinischen Auswirkungen auf den Patienten sofort zu analysieren. Insbesondere epidurale Zementleckagen müssen unter Berücksichtigung ihres Ausmaßes und der durch sie verursachten Spinalkanalstenose sofort hinsichtlich des Erfordernisses einer intraoperativen Dekompression überprüft werden. Intradiskale Leckagen müssen hinsichtlich der Möglichkeit einer subsequenten leckageinduzierten Anschlussfraktur analysiert werden, um ggf. eine Schutzaugmentation des Anschlusswirbelkörpers durchführen zu können. Endovaskuläre Leckagen sollten hinsichtlich einer unmittelbaren systemischen Auswirkung in direkter Kommunikation mit dem Anästhesisten besprochen werden. Außerdem ist aus unserer Sicht auch bei zunächst klinisch inapparentem Verlauf eine postoperative Computertomografie des Thoraxes durchzuführen, um spätestens hier evtl. größere dislozierte Zementmengen zu erkennen und entsprechend prophylaktisch reagieren zu können.

\section{Tipps und Tricks}

Insbesondere bei der Frakturbehandlung stellt sich die Frage nach dem Ausmaß der notwendigen intraoperativen Reposition. Einerseits soll die Reposition bestmöglich durchgeführt werden, andererseits soll im strukturschwachen Knochen die Instrumentierung nicht über das nötige Ausmaß hinaus zusätzlich beansprucht werden. 


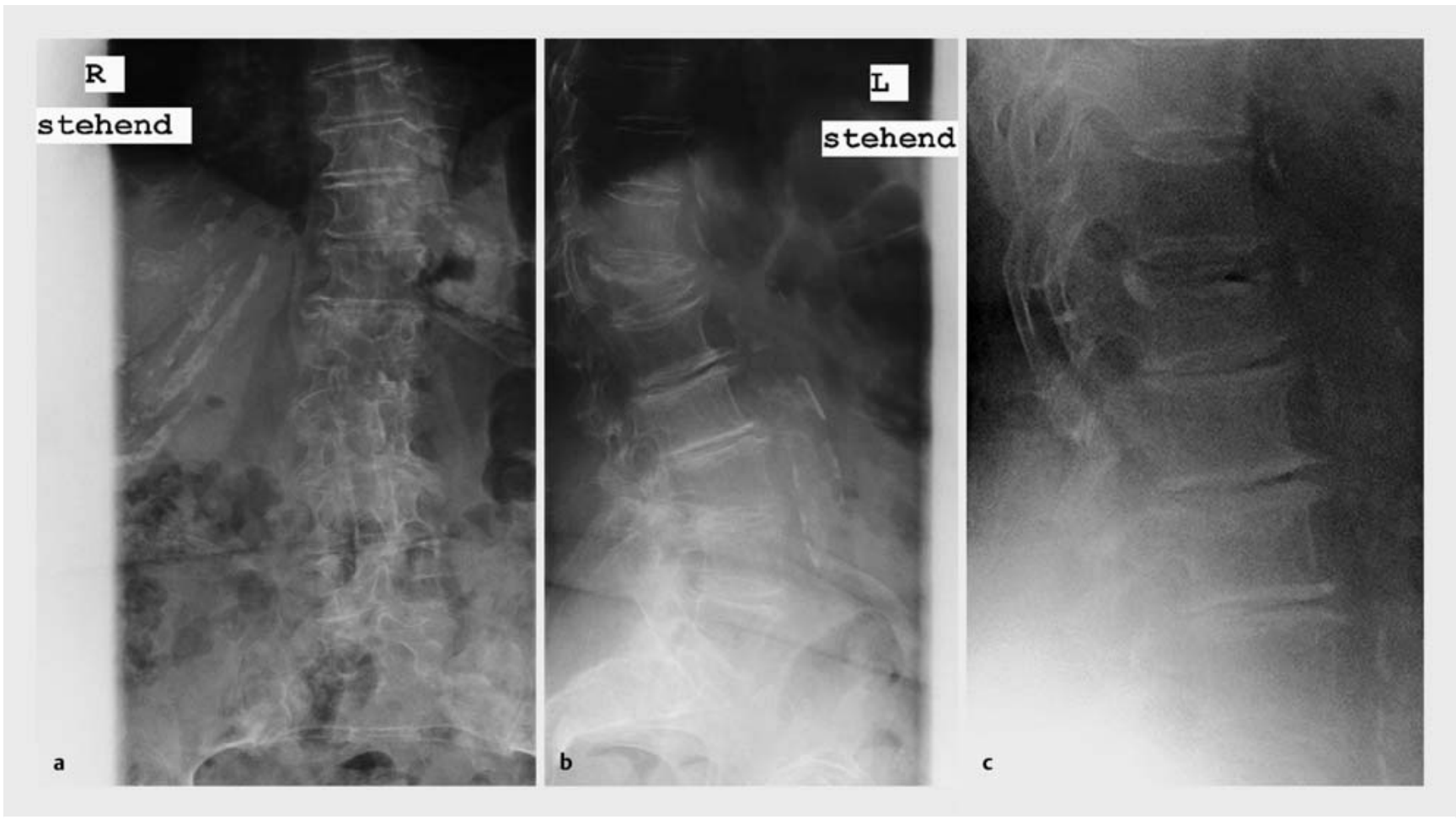

- Abb. 5 79-jährige Patientin: Konventionelle Röntgenaufnahmen in der a.-p. (a) und seitlichen (b) Ebene jeweils stehend bei einer Th-XII-Fraktur im strukturschwachen Knochen mit deutlicher segmentaler Kyphosierung. Seitliche Hypomochlionaufnahme in Rückenlage mit spontaner Relordosierung (c).

\section{Merke}

Für die Versorgung frischer osteoporotischer Frakturen empfehlen wir daher die präoperative Durchführung einer Hypomochlionaufnahme in seitlicher Einstellung, wobei dem in Rückenlage befindlichen Patienten das Hypomochlion (z. B. weiches Zylinderkissen) unmittelbar in Projektion auf den frakturierten Wirbelkörper untergeschoben wird.

Nach ca. 10 Minuten entspannter Ruhezeit kann dann eine seitliche Röntgenaufnahme durchgeführt werden. Die Spontanreposition, die sich hier einstellt, ist dann Vorgabe der intraoperativ anzustrebenden Reposition $(\triangleright$ Abb. 5). Eine Reposition unter dem erreichten und in der Hypomochlionaufnahme dargestellten Ausmaß ist nicht erforderlich. Eine Reposition über das in der Hypomochlionaufnahme dargestellte Ausmaß hinaus könnte die Instrumentierung überdurchschnittlich belasten und zu einem Sekundärversagen trotz Zementaugmentation führen.

Besonderes Augenmerk sollte bei der perkutanen, also gedeckten Technik, auf die Möglichkeit eines retrograden Zementaustritts in den Schraubenkopf gelegt werden. Dies kann intraoperativ besonders unangenehm werden, weil der Schraubenkopf nicht unmittelbar eingesehen werden kann und somit eine retrograde Zementleckage in den Schraubenkopf bis zur Polymerisation des Zements zunächst oft unerkannt bleibt. Erst wenn sich dann die Insertion des Stabes als technisch unmöglich erweist, wird die Verlegung des Schraubenkopfs mit Zement schließlich erkannt ( $\triangleright$ Abb. 6). Dem kann von vornherein entgegengewirkt werden, indem mit der Diskonnektion der Zementinjektionskanüle vom Schraubenkopf für ca. 2-3 Minuten nach Beendigung der Zementinjektion gewartet wird.

Insbesondere bei multilokulärer Pathologie empfehlen wir zusätzlich die grundsätzliche Entnahme einer Knochenbiopsie, z. B. zum Ausschluss eines multiplen Myeloms.

\section{Revisionsverhalten zementierter Pedikelschrauben}

Hinsichtlich des Verhaltens zementaugmentierter Pedikelschrauben im Revisionsfall führten wir eine In-vitroStudie durch. An humanen osteoporotischen Brust- und Lendenwirbelkörpern erfolgte eine transpedikuläre Instrumentierung mit kanülierten fenestrierten Pedikelschrauben der Dimension 6,5 $\times 45 \mathrm{~mm}$. Pro Schraube wurden jeweils $1,5 \mathrm{ml}$ PMMA-Zement injiziert. Nach Zementpolymerisation (30 Minuten nach Injektion) wurde das 


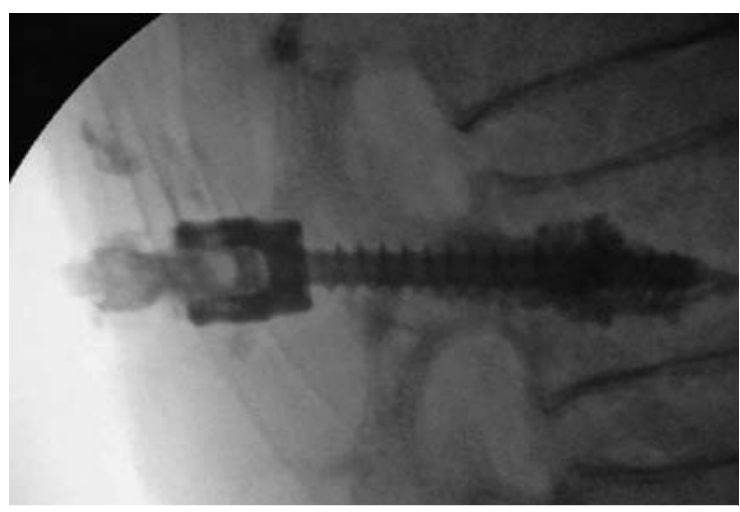

- Abb. 6 Intraoperative Bildverstärkeransicht in der seitlichen Ebene: retrograde Zementleckage in den Schraubenkopf unmittelbar nach Schraubenaugmentation.

zum Ausdrehen der Schrauben erforderliche Drehmoment bestimmt. Sowohl die Schraubenimplantation als auch die Schraubenexplantation erfolgten unter Bildverstärkerkontrolle, um mögliche Zementmitbewegungen während der Schraubenexplantation erkennen zu können.

Das Ergebnis der Untersuchung zeigte ein insgesamt völlig problemloses Explantationsverhalten der Pedikelschrauben: Bei einer durchschnittlichen Knochendichte von $0,602 \mathrm{~g} / \mathrm{cm}^{3}$ war das mittlere Explantationsdrehmoment mit 0,49 Nm sehr gering. Sichtbare Zementbewegungen waren nicht zu erkennen.

\section{Merke}

Als Fazit dieser Untersuchung lässt sich festhalten, dass die Revisionscharakteristik von zementaugmentierten Schrauben unproblematisch ist, auch in der Gegenwart einer schweren Osteoporose.

Die flügelartigen Zementverbindungen zwischen dem Schraubeninneren und dem umgebenden Knochengewebe sind zerbrechlich genug, um beim Auftreten eines geringen Extraktionsdrehmoments abzubrechen und die Schraube freizugeben. Dieses Ergebnis wurde auch durch weitere Arbeitsgruppen bestätigt $[13,14]$.

\section{Schlussfolgerungen}

- Eine Zementaugmentation von Pedikelschrauben kann die Rate an Schraubenlockerungen und sekundärer Auswanderung von Implantaten deutlich reduzieren.

- Die Leckagerate bei der Zementaugmentation von Pedikelschrauben zeigt einen durchschnittlichen Anteil von $20 \%$.

- Die Revision zementaugmentierter Schrauben ist im Allgemeinen unproblematisch.
- Bei der Frakturbehandlung ist eine optimierte Reposition gegenüber der maximal möglichen Reposition eines der hauptsächlichen Ziele.

\section{Interessenkonflikt}

Vortragshonorare/Medical Advisory Boards: Aesculap, AOSpine, Biomet, DePuySynthes, EUROSPINE, Integra, Magnifi, Medtronic, Spontech, Vexim

Über die Autoren

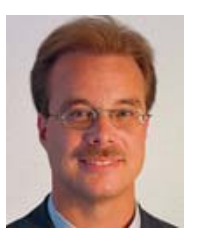

\section{Thomas R. Blattert}

Prof. Dr. med. Th. R. Blattert. Studium der Humanmedizin in Ulm, St. Gallen und Boston. Seit 2010 Ärztliche Leitung der Abteilung Wirbelsäulenchirurgie und Traumatologie an der Orthopädischen Fachklinik Schwarzach. Generalsekretär der EUROSPINE. Vorstandsmitglied der DWG. Past-Chairman des deutschen Councils der AOSpine. Gastprofessor des Xi'an Jiaotong University Medical College Honghui Hospital, China.

\section{Korrespondenzadresse}

Prof. Dr. med. Thomas R. Blattert

Chirurg, Orthopäde und Unfallchirurg, Spezielle Unfallchirurgie

Chefarzt Wirbelsäulenchirurgie und Traumatologie

Orthopädische Fachklinik Schwarzach

Dekan-Graf-Straße 2-6

94374 Schwarzach

thomas.blattert@ofks.de

\section{Literatur}

[1] Tolunay T, Başgül C, Demir T et al. Pullout performance comparison of pedicle screws based on cement application and design parameters. Proc Inst Mech Eng H 2015; 229: 786-793

[2] Shea TM, Laun J, Gonzalez-Blohm SA et al. Designs and techniques that improve the pullout strength of pedicle screws in osteoporotic vertebrae: Current status. BioMed Research International 2014; Article ID 748393

[3] Kolb JP, Weiser L, Kueny RA et al. Zementaugmenation an der Wirbelsäule. Was ist biomechanisch zu beachten? Orthopäde 2015; 44: 672-680

[4] Elder BD, Lo SF, Holmes C et al. The biomechanics of pedicle screw augmentation with cement. Spine J 2015; 15: 14321445

[5] Schnake KJ, Hahn P, Franck A et al. Development of a classification system (OF-classification) and of a score for therapeutic decision making (OF-score) for osteoporotic thoracolumbar fractures. Eur Spine J 2013; 22: S2590

[6] Blattert TR, Schnake KJ, Gonschorek O et al. Guidelines for non-surgical and surgical management of osteoporotic vertebral body fractures. Eur Spine J 2015; 24: S703

[7] Hu M, Wu H, Chang M et al. Polymethylmethacrylate augmentation of the pedicle screw: the cement distribution in the vertebral body. Eur Spine J 2011; 20: 1281-1288

[8] Blattert T, Katscher S, Josten C. Perkutane Techniken an der Brust- und Lendenwirbelsäule. Unfallchirurg 2011; 114: 17-25 
[9] Koreckij T, Park D, Fischgrund J. Minimally invasive spine surgery in the treatment of thoracolumbar and lumbar spine trauma. Neurosurg Focus 2014; 37: E11

[10] Blattert TR, Glasmacher S, Siekmann H et al. Combined vertebral stabilization by means of cement-augmented posterior instrumentation and balloon-kyphoplasty in osteoporotic burst fractures. Eur Spine J 2009; 18: S456-S457

[11] Janssen I, Ryang Y, Gempt J et al. Risk of cement extravasation and pulmonary embolism by bone cement augmented pedicle screw fixation of the thoracolumbar spine. Eur Spine J 2015; 24: $\$ 2623$

[12] Mueller J, Baldauf J, Marx S. Cement leakage in pedicle screw augmentation: a prospective analysis of 98 patients and 474 augmented pedicle screws. J Neurosurg Spine 2016; 25: $103-$ 109
[13] Blattert TR, Glasmacher S, Riesner H] et al. Revision characteristics of cement-augmented, cannulated fenestrated pedicle screws in the osteoporotic vertebral body: a biomechanical in vitro investigation. J Neurosurg Spine 2009; 11: 23-27

[14] Bullmann V, Schmoelz W, Richter M et al. Revision of cannulated and perforated cement-augmented pedicle screws: a biomechanical study in human cadavers. Spine (Phila Pa 1976) 2010; 35: E932-E939

Bibliografie

DOI http://dx.doi.org/10.1055/s-0043-101394

OP-JOURNAL 2017; 33: 4-10 @ Georg Thieme Verlag KG Stuttgart · New York ISSN 0178-1715 